\title{
Use of osteosynthesis titanium micro-plates in rhinoplasty
}

\author{
Rui Xavier ${ }^{1}$, Hugo Amorim ${ }^{1}$, Dirk Jan Menger ${ }^{2}$, Henrique Carvalho ${ }^{1}$, and Jorge Spratley ${ }^{3}$ \\ ${ }^{1}$ Universidade do Porto Instituto de Ciencias Biomedicas Abel Salazar \\ ${ }^{2}$ University Medical Center Utrecht \\ ${ }^{3}$ Universidade do Porto Faculdade de Medicina
}

November 10, 2020

\begin{abstract}
In reconstructive rhinoplasty, the nasal framework must be securely fixated to the facial bony skull. We present a surgical technique to fixate the nasal L-strut to the facial skull by using exclusively titanium L-shaped micro-plates in cases where the anterior nasal spine and the nasal bones cannot be used
\end{abstract}

Use of osteosynthesis titanium micro-plates in rhinoplasty

Rui Xavier (1,2), Hugo Amorim (1), Dirk-Jan Menger (3), Henrique Cyrne de Carvalho (2), Jorge Spratley (4)

1 Hospital Luz Arrabida, Porto, Portugal

2 Instituto de Ciências Biomédicas Abel Salazar, Universidade do Porto, Porto, Portugal

3 University of Utrecht, Utrecht, The Netherlands

4 Faculdade de Medicina da Universidade do Porto, Centro Hospitalar e Universitário S. João and Centro de Investigação em Tecnologias e Serviços de Saúde (CINTESIS), Porto, Portugal

Ethical considerations:

This manuscript complies with all current ethical requirements. The patient whose clinical case and photos illustrate this manuscript was informed of this manuscript and signed a written consent for his case and photos to be published.

The authors declare that they have no conflicts of interest to disclose

Corresponding author:

Rui Xavier

rjxavier65@gmail.com

Department of Otorhinolaryngology, Hospital Luz Arrabida

Praceta Henrique Moreira 150

4400-346 VNGaia - Portugal

Abstract

Objectives 
To demonstrate that solid fixation of the nasal L-strut is possible even in the most difficult clinical scenario of reconstructive rhinoplasty ensuring good long-term results.

\section{Settings}

In most cases of reconstructive rhinoplasty, the nasal framework has to be securely fixated to the facial bony skull. Solid points on the facial skull for suturing the nasal framework are usually available but, occasionally, may be absent. In these circumstances, alternative solutions must be used.

Methods

We present a surgical technique to fixate the nasal L-strut to the facial skull by using exclusively titanium Lshaped micro-plates in cases where the anterior nasal spine and the nasal bones cannot be used for providing fixation points.

The case of a patient with severe dysmorphia of the nose is presented as illustrative of the technique. Four L-shaped titanium micro-plates usually utilized for osteosynthesis were used to fixate superiorly extended spreader grafts and a caudal septal extension graft to the adjacent bony skull.

The reporting methods do not apply for this manuscript.

Results

This combined caudal and dorsal fixation of the nasal framework to the facial bony skull by using titanium micro-plates has not been previously described. Nevertheless, we were able to create a solid framework for the nasal pyramid, ensuring a stable long-term result.

Conclusions

Even in the most difficult clinical scenario of reconstructive rhinoplasty a solid fixation of the nasal L-strut is possible, by using titanium micro-plates screwed to the facial bony skull. This fixation has proved to be stable, providing good long-term results.

Keywords: Rhinoplasty, Titanium micro-plates, Nasal L-strut fixation, Nasal framework, Reconstructive rhinoplasty

Key Clinical Message

Even in the most difficult clinical scenario of reconstructive rhinoplasty a solid fixation of the nasal L-strut is possible. Fixation by using titanium micro-plates screwed to the facial skull has proved to be stable providing good long-term result

\section{Introduction}

The high degree of expectancy of the surgical outcome of rhinoplasty from both the patient and the surgeon makes every single rhinoplasty case a challenge. Reconstructive rhinoplasty is amongst the most difficult cases, as rebuilding the nasal framework has the extra burden of providing a solid midline structure upon which the nasal pyramid is erected. Usually there are solid points on the bony facial skull for suturing the nasal framework. In rare cases those solid points for suturing cannot provide strong support and alternative solutions have to be used. A surgical technique for fixation of the L-strut to the facial skull using exclusively titanium micro-plates is described. An illustrative clinical case in which this technique was used is presented.

\section{Clinical Case}

The patient is a 28 years old male from African descent. In his medical history there were no known history of nasal trauma or of nasal or systemic diseases. The patient had no nasal airway obstruction but wanted to undergo surgery to achieve an aesthetic improvement of the nose.

Nasal analysis revealed an absent nasal dorsum with only the tip protruding from the face (Fig.1). The nasal bones and ascendant processes of the maxilla were flat, with no convexity and not sagittally protruding from 
the facial plane. The middle third of the nose was hypoplastic due to underdeveloped septal and upper lateral cartilages. The nasal tip was wide and under projected, with insufficient support. There was retraction of the caudal septum and the anterior nasal spine could not be palpated. The nasal cavities had reasonable width on both sides and the septum was at the midline.

A CT scan of the nose of the patient demonstrated a hypoplastic anterior nasal spine (Fig.2a). The nasal bones were present though hypoplastic and not protruding from the face as shown on the CT 3D reconstruction (Fig.2b).

The patient wanted to undergo rhinoplasty for an aesthetic improvement of the nose. He requested an increase in tip projection and in tip definition, while preserving the ethnic features of his nose. Most importantly, the patient desired to have a protruding nasal dorsum. Given the specific features of the patient's nose, the surgical plan for the rhinoplasty was thoroughly discussed with the patient.

\section{Surgical Report}

Fascia from pectoralis major muscle and costal cartilage from the $7^{\text {th }}$ rib were harvested. An extended open rhinoplasty approach was used. The quadrangular septal cartilage was hypoplastic, causing retraction of the caudal septum and leading to the low middle third of the nose. The anterior nasal spine was barely palpable. Soft tissues were elevated from the anterior surface of the premaxilla in order to expose the bone of the premaxilla. Septal mucoperichondrial flaps were raised bilaterally. The upper lateral cartilages were divided from the dorsal septum. The nasal bones and ascending processes of the maxilla were widely exposed up to the glabella.

Median and paramedian osteotomies were performed in order to remove a wedge of the nasal bones at the midline. The gap thus created was used to accommodate superiorly (and inferiorly) extended spreader grafts. No other osteotomies were performed as not to destabilize the nasal bones and ascending processes of the maxilla.

Extended spreader grafts from costal cartilage were docked in the gap created between the nasal bones and positioned anteriorly in relation to the plane of the nasal bones. The cephalic margins of the extended spreader grafts were positioned to abut the frontal bone, in the glabella. Titanium micro-plates bent in order to create L-shaped micro-plates were used to fixate these grafts to the flat nasal bones. A micro-screw (1.5 $\mathrm{mm}$ wide, $3 \mathrm{~mm}$ long) was screwed to fixate the horizontal arm of the L-shaped titanium plate to the nasal bone and another micro-screw was screwed to fixate the vertical arm of the titanium plate to the lateral surface of the cephalic region of the spreader graft (Fig.3). This attachment was done to both extended spreader grafts.

A septal extension graft was created from two pieces of costal cartilage sutured one to the other, with the concave sides facing inside. This was done to prevent any deviation of the nasal tip off the midline due to cartilage warping. This double-layered cartilage was then fixated to the premaxilla using two L-shaped titanium micro-plates. A micro-screw was screwed to fixate the horizontal arm of the L-shaped titanium plate to the maxillary bone close to the anterior nasal spine and another micro-screw was screwed to fixate the vertical arm of the titanium plate to the lateral surface of the septal extension graft (Fig.4a) This attachment was done on both sides of the septal extension graft.

The caudal region of the two extended spreader grafts was then sutured to the anterior region of the septal extension graft with a non-resorbable suture (Fig.4b,c).

Figure 5 illustrates how the extended spreader grafts and the caudal extension graft were fixated to the bony skull in order to provide a solid nasal framework. The upper lateral cartilages were sutured to the spreader grafts and the medial crura of the lower lateral cartilages to the septal extension graft in a tongue-in-groove fashion. Domal sutures and an interdomal suture were used. A tip and an infratip graft were both sutured in place. Free diced cartilage was placed in the radix in order to smooth the contour of the nasofrontal angle as well as between the two extended spreader grafts to fill the space between the grafts. A dorsal onlay graft 
from costal cartilage was sutured over the nasal dorsum, to increase dorsal projection. A layer of pectoralis major fascia was placed over the nasal dorsum, in order to smooth the contour of the nasal dorsum.

A septal mattress suture was used and the marginal and columellar incisions were sutured using a fast resorbing suture material. An external cast with a thermic splint was used. Intra-nasal packing was not used.

The patient was discharged on the day after surgery. He was prescribed oral amoxicillin-clavulanic acid for 8 days, paracetamol as required, and was instructed to apply an ointment with gentamicin and dexamethasone over the sutures twice a day and to do intranasal lavages with saline for eight weeks. The external cast was removed 10 days after surgery.

Two years after surgery the rebuilt nasal framework remains stable and securely attached to the facial skull by the four L-shaped titanium micro-plates. No signs of infection or of extrusion of the screws have occurred during this time. The pre-operative photos and the two years post-operative photos of the patient are shown in Figures 1a,b,c,d and 6a,b,c,d.

Discussion

Every rhinoplasty case is a challenge. The degree of sophistication of rhinoplasty has reached to a point where results are expected to be perfect, leaving little room to a suboptimal outcome. Reconstructive rhinoplasty is amongst the most difficult rhinoplasty cases, as the nasal framework has to be significantly reinforced or even completely rebuilt. Upon this rebuilt nasal framework the nasal pyramid can be erected to provide shape to the external nose and an efficient airway.

Usually there are solid bony points for suture fixation of the new nasal framework, namely the anterior nasal spine for caudal fixation and the nasal bones and ascending processes of the maxilla for cephalic fixation of the nasal L-strut. These can be drilled to make holes where suture material can be passed to strongly secure the cephalic part of the nasal L-strut. Alternatively, the transcutaneous transosseous cerclage suture described by Haak et al $(1,2)$ can also be used for fixating the dorsal part of the nasal L-strut to the adjacent facial bones. A straight osteotome or a powered instrument can be used to create a notch in the anterior nasal spine where the caudal and posterior part of the L-strut can be slipped in or a hole may be drilled on the anterior nasal spine in order to suture the caudal margin of the L-strut. In this way, a nasal framework solidly secured to the facial skull can be achieved, ensuring a stable long-term outcome.

In rare instances these traditional solutions can not be used. This was the case of the patient that we present. Due to the shape and orientation of the nasal bones and ascending processes of the maxilla, which were not sagittally protruding from the plane of the face, these could not be used for the traditional types of suture attachment of the nasal L-strut. Moreover, due to the almost complete absence of the anterior nasal spine, also the usual caudal fixation of the nasal framework could not be used. Therefore, alternative solutions had to be found. We used four titanium micro-plates bent into a L-shape for securing the nasal framework to the facial skull.

The use of titanium micro-plates to fixate the caudal part of the nasal framework to the maxilla in cases of absent anterior nasal spine has been described $(1,3)$, but using titanium micro-plates to fixate superiorly extended spreader grafts to the nasal bones and adjacent ascending processes of the maxilla has not, to our knowledge, been previously described.

Mittermiller et al (3) used titanium plates in the premaxilla for suturing the caudal septum in cases of absent anterior nasal spine. Haaket al (1) used titanium plates for fixating a transposed anterior nasal spine to the midline or to substitute the anterior nasal spine. In the nasal dorsum titanium plates have been used to fixate the dorsal septum to the nasal bones in cases of traumatic fractures of the nasal septum (4) and to fixate fragments of fractured nasal bones or ascending processes of the maxilla (4). Titanium microplates have also been used in the nose as stents sutured to the undersurface of costal cartilage grafts to prevent warping of the grafts or sutured as stents to the L-strut to stabilize the anterior septal angle $(3,5)$.

Titanium plates have been widely used in maxillo-facial surgery with not significant host reaction or ex- 
trusion. Therefore, we felt confident to use titanium micro-plates in the nose. The risk of infection of these implanted materials was low due to the fact that the micro-plates and the screws were not in contact with the nasal airway or with the nasal mucosa at any stage of the surgery or afterwards. In the clinical case that we report, there was no infection in the two years that have passed since the rhinoplasty.

In our clinical case both the cephalic and the caudal margins of the newly created nasal framework were fixated to the face by titanium micro-plates. This has not been previously reported. We bent the titanium micro-plates as to mold each one with the L-shape that would suit our fixation requirements. Differently from previous descriptions of titanium plates used in the nose $(1,3)$, we did not suture the grafts to the micro-plates; instead, we screwed the micro-plates to the bone and to the costal cartilage grafts with microscrews. Screwing these titanium micro-plates to grafts made from strong costal cartilage led the impression of a stable attachment. Nevertheless, after being screwed, the extended spreader grafts could be gently tilted as needed in order to make fine adjustments of the angulation of these grafts and to set the ideal relative position between the extended spreader grafts and the septal extension graft. The length of the micro-screws that we used $(3 \mathrm{~mm})$ was very appropriate to secure the titanium micro-plate to the bone without overpassing the depth of the bone. Some over-pass of the screws on the extended spreader grafts did happen, but the excess of the length of the screws was well concealed between the two spreader grafts.

An extended open approach was necessary to allow drilling and screwing the titanium micro-plates to the facial skull. This may have led to more significant local edema, but did not prevent the patient from being discharged on postoperative day one.

The specific anatomical features of the patient led us to use this surgical solution. The ethnic African nose of the patient is not a justification for the complete absence of nasal dorsum, and there was no known reason for that. No systemic or local disease was present. The blood tests conducted were all normal and the patient was healthy. He did not report any previous nasal trauma, but perhaps some form of trauma had occurred during childhood. This could have led to the abnormal shape and position of the nasal bones and ascending processes of the maxilla as well as to some resorption or under-development of the anterior nasal spine and cartilaginous middle third of the nose. Remarkably, despite the absence of nasal dorsum, the patient had no complaints of nasal airway obstruction.

Other surgical solutions could have been used to address this patient. As the patient had no nasal airway obstruction the option of using superiorly and inferiorly extended spreader grafts positioned anteriorly to increase the height of the nasal dorsum plus a cartilaginous dorsal onlay graft could have been disfavored to a simpler cantilevered dorsal onlay graft. However, we thought that the necessary height of this onlay piece of cartilage could have created a heavy burden over the underdeveloped cartilages of the middle third of the nose eventually creating nasal airway obstruction. Composite reconstruction of the nasal dorsum using spreader grafts normally positioned together with a dorsal onlay graft of glued diced cartilage or diced cartilage in fascia could have been another option, but we thought that expecting a strong new dorsum from this diced cartilage graft in a patient with no nasal dorsum and with thick skin would be over optimistic.

Fascia from pectoralis major muscle was harvested from this patient, as previously described (6). This patient, from African descent, had thick skin. Nevertheless, we felt more confident to use a layer of fascia over the rebuilt nasal dorsum to prevent any irregularity of the grafts to become apparent in the long-term.

The surgical solution that was taken has proved to remain stable over time, as shown by the two-years postoperative photos of the patient. We are confident that the strong nasal structure that was created by using costal cartilage grafts screwed to the facial bones will stand the test of time and will ensure a stable long-term result. No signs of local reaction or extrusion of the titanium micro-plates or of the screws have occurred during these two years. Nevertheless, we will continue to observe the patient as we believe that rhinoplasty is a life-long commitment between surgeon and patient.

\section{Conclusions}

A distinctive feature of modern rhinoplasty is the ability to incorporate expertise and solutions from other 
medical fields into nasal surgery, in the continuous search for improving the results. When the traditionally used facial bony structures cannot be used for suture fixation of the nasal L-strut other solutions have to be used, in order to achieve the ultimate goal of a solid nasal framework that will ensure a stable long-term outcome. We describe a new way of fixating the nasal framework to the facial skull by using exclusively titanium micro-plates. This surgical solution, though not frequently necessary, can be incorporated in the armamentarium of the rhinoplasty surgeon.

The authors declare that they have no conflicts of interest to disclose.

Author contributions:

Rui Xavier: conception and design, acquisition of data, analysis and interpretation of data, drafting the manuscript, revising it critically and given final approval of the version to be published.

Hugo Amorim: conception and design, acquisition of data, analysis and interpretation of data.

Dirk-Jan Menger: conception and design, revising the manuscriopt critically and given final approval of the version to be published.

Henrique Cyrne de Carvalho: conception and design, revising the manuscriopt critically and given final approval of the version to be published.

Jorge Spratley: conception and design, interpretation of data, drafting the manuscript, revising it critically and given final approval of the version to be published.

Acknowledgement statement:

None

References

1. Haack S, Hacker S, Mann S, Gubisch W. Bony fixation of the nasal framework. Facial Plast Surg 2019; 35:23-30

2. Rezaeian F, Gubisch W, Janku D, Haack S. New suturing techniques to reconstruct the keystone area in extracorporeal septoplasty. Plastic and Reconstructive Surgery 2016; 138(2):374-382

3. Mittermiller P, Sheckter C, Most S. Efficacy and Safety of Titanium Miniplates for Patients Undergoing Septorhinoplasty. JAMA Facial Plast Surg 2018; 20(1):82-84

4. Manuel A, Fishman J, Majumdar A, Draper M. Titanium mini-plate for securing septal cartilage - a case of high-impact nasal trauma. Ann R Coll Surg Engl 2009; 91:433-434

5. Eren F, Öksüz S, Melikoğlu C, et al . Saddle-nose deformity repair with microplate-adapted costal cartilage. Aesthetic Plast Surg 2014; 38(4):733-741

6. Xavier R. Pectoralis major fascia in rhinoplasty. Aesthetic Plast Surg 2015; 39(3):300-305

Figure legends

Fig.1a,b,c,d Preoperative photos of the patient

Fig.2a,b 3D reconstruction from the CT scan showing the abnormal nasal bone structure of the patient (a) and the flat nasal bones $(\mathrm{b})$

Fig.3a,b,c Titanium micro-plate screwed to the nasal bone and to the cephalic margin of the spreader graft on each side

Fig.4a,b,c Titanium micro-plate screwed to the premaxilla and to the caudal part of the caudal extension graft on each side of the midline (a) and fixation of the extended spreader grafts to the caudal extension graft $(b, c)$

Fig.5 Illustration of the nasal framework fixation to the bony skull with titanium micro-plates

Fig. 6a,b,c,d Two years postoperative photos of the patient 

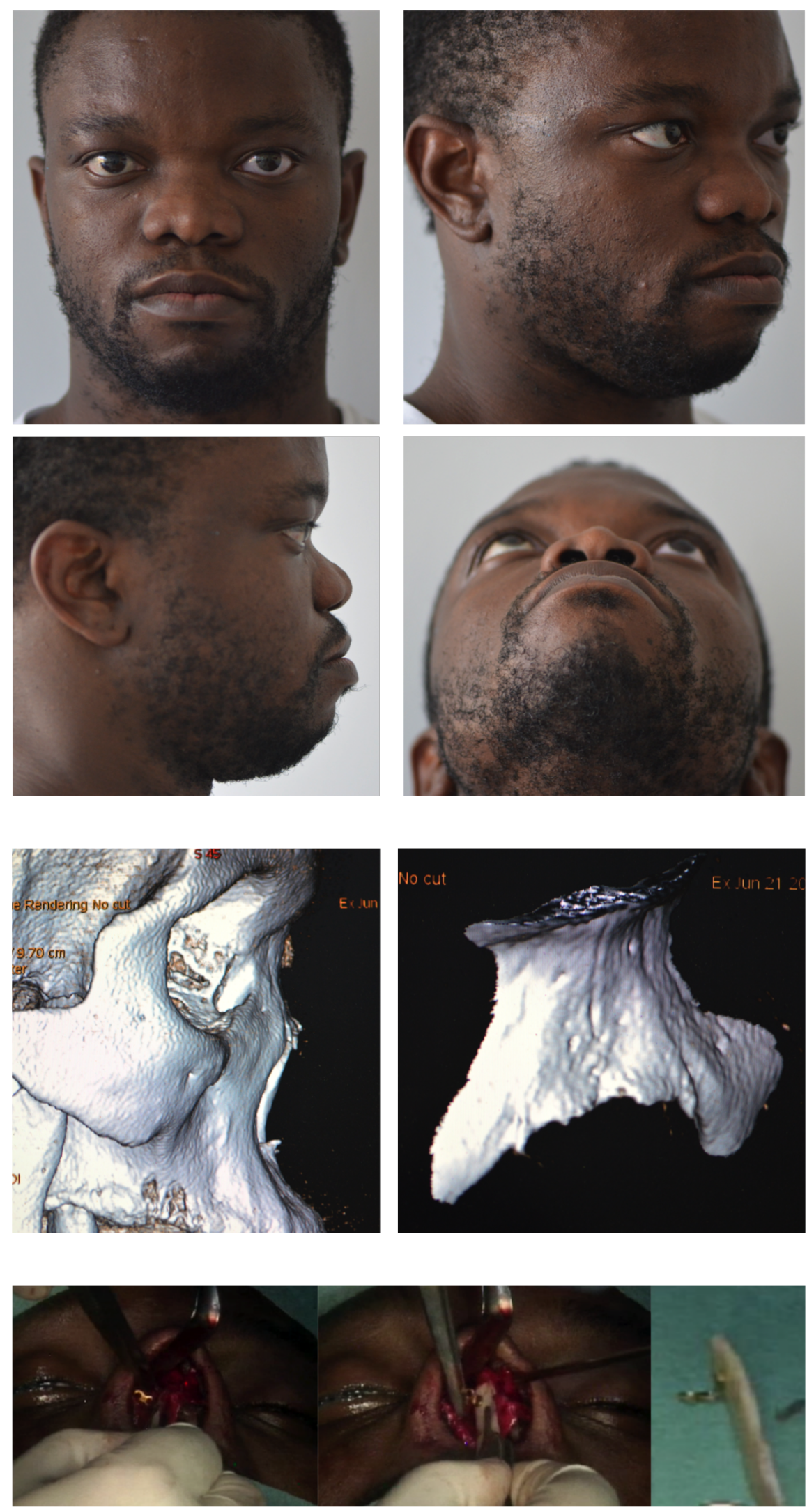

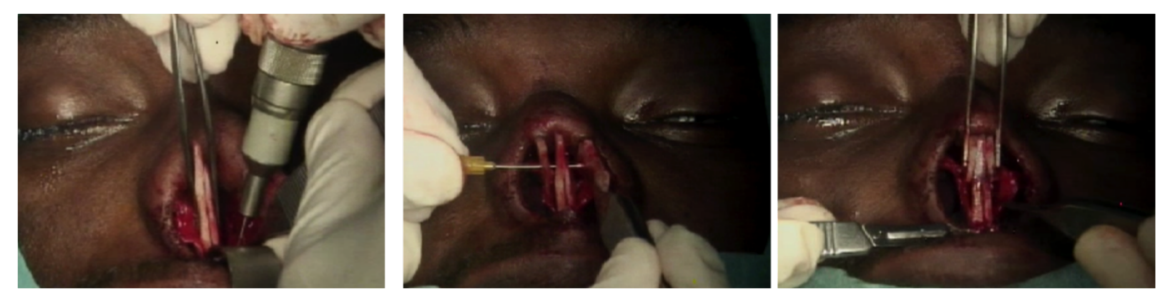

Fremberar 

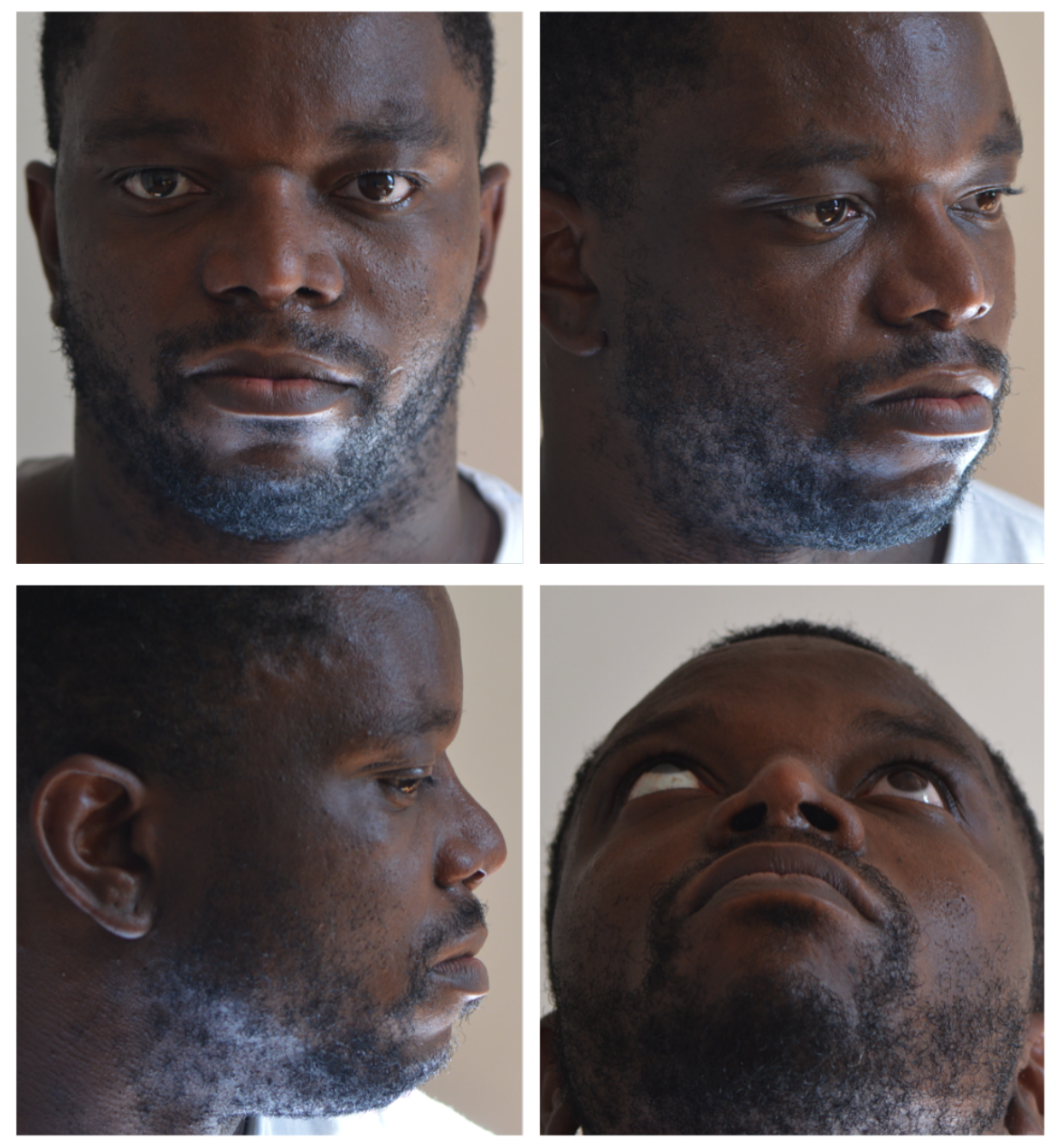